APLICAÇÃO DE UM JOGO EDUCATIVO COMO RECURSO PARA O ENSINO DE QUÍMICA

\title{
APPLICATION OF A EDUCATIONAL GAME AS RESOURCE FOR TEACHING CHEMISTRY
}

\author{
SILVA, C. K O ${ }^{1 *}$; NOGUEIRA, J. P. A ${ }^{2}$; SOUZA, H. Y. S. ${ }^{3}$ \\ ${ }^{1,3}$ Secretaria de Estado da Educação e da Cultura do RN, SEEC RN, Brasil \\ Centro Administrativo do Estado, BR 101-km 0, Lagoa Nova-Natal/RN, CEP: 59064-901, Natal - RN, Brasil \\ (fone: +55 84 3232-1304)
}

${ }^{2}$ Universidade Federal do Rio Grande do Norte, Engenharia de Petróleo, Campus da UFRN, Lagoa Nova, CEP: 59.078-970, Natal-RN, Brasil (fone: +55 84 32153904)

\author{
* Autor correspondente \\ e-mail: celyna_ceu@hotmail.com
}

Received 23 November 2011; received in revised form 05 December 2011; accepted 07 December 2011

\begin{abstract}
RESUMO
A maioria dos alunos do ensino médio apresenta certa aversão à disciplina de Química. Isso ocorre, provavelmente, devido à complexidade dos fenômenos envolvidos nas transformações químicas e a pouca interdisciplinaridade e/ou transdisciplinaridade em relação com o cotidiano do aluno e com as outras disciplinas. Por isso, o professor deve encontrar alternativas pedagógicas que auxiliem no processo de ensinoaprendizagem de Química. O desenvolvimento de estratégias modernas e simples utilizando experimentos, jogos e outros recursos didáticos, é recomendado para dinamizar o processo de aprendizagem em Química. $O$ objetivo deste trabalho foi empregar um jogo como recurso didático para facilitar o processo de ensinoaprendizagem de Química. O jogo utilizado foi o Sudoku, sendo a versão empregada denominada Sudokímica. O material foi aplicado em três turmas de ensino médio de uma escola pública de Natal-RN, Brasil. O recurso didático aplicado obteve resultados satisfatórios, pois tanto facilitou como integrou os conhecimentos científicos com os conhecimentos do cotidiano dos alunos. Isto pode ser concluído que o jogo, Sudokímica, pode ser considerado como uma boa alternativa de material suplementar no processo de ensino-aprendizagem de química por resoluções de problemas.
\end{abstract}

Palavras-chave: Jogo educativo; ensino-aprendizagem.

\begin{abstract}
Most of the high school students have an aversion to the subject of chemistry. This fact is, probably, due to the complexity of the phenomena involved in chemical transformations and little interdisciplinarity and / or transdisciplinarity in relation to the daily life of students and with other subjects. Therefore, the teacher must find ways to assist the pedagogical teaching-learning process in chemistry. The development of modern and simple strategies, using experiments, games and other teaching resources, is recommended to streamline the process of learning chemistry. The aim of this work was to employ a game as a didactical resource to facilitate the teaching-learning process in chemistry. The game used was Sudoku, and the version employed was called Sudokímica. The material was applied in three high school classes of a public school in Natal-RN, Brazil. The didactical resource was successful in the sense of facilitating and integrating the scientific and daily life knowledge of the students. It can be concluded that the game, Sudokímica can be considered good alternative to supplementary material in the teaching-learning process in chemistry through for resolution of problems.
\end{abstract}

Keywords: Educational game, teaching-learning.

PERIÓDICO TCHÊ QUÍMICA • www.periodico.tchequimica.com • Vol. 9 N. 17. - ISSN 1806-0374 (impresso) • ISSN 1806-9827 (CD-ROM) • ISSN 2179-0302 (meio eletrônico) 
INTRODUÇÃO

O dia-a-dia do professor é bastante dinâmico e requer sempre a execução de trabalhos didáticos que estimulem 0 aprendizado. Uma alternativa para a dinamização das aulas é variar as técnicas de ensino empregadas.

Quando se cria ou se adapta um jogo ao conteúdo escolar, ocorre o desenvolvimento de habilidades que envolvem o indivíduo em todos os aspectos: cognitivos, emocionais e relacionais. Segundo Vygotsky (1989), os jogos estimulam a curiosidade, a iniciativa e a autoconfiança, aprimoram o desenvolvimento de habilidades linguísticas, mentais e de concentração e exercitam interações sociais e o trabalho em equipe.

Muitas pesquisas envolvendo jogos foram publicadas no sentido de auxiliar o professor de Química na sua prática pedagógica, tais como: Super Trunfo de Química (GODOI et al., 2010), Soletrando o Br-As-I-L com Símbolos Químicos (MARISCAL; IGLESIAS, 2009), Suequímica (SANTOS; MICHEL, 2009), Ludo Químico (ZANON, 2008), Memória Orgânica (WATANABE; RECENA, 2008), Bingo Químico, Trilha Química (SANTOS et al., 2008), Clube da Química, Dominó dos Plásticos (BARROS et al., 2009).

Todas essas pesquisas foram pensadas na tentativa de encontrar alternativas que perpassem as barreiras do ensino tradicionalista, voltado para a utilização do livro didático sem nenhuma relação com o cotidiano do aluno, o que cria muitas vezes uma barreira intransponível entre o conteúdo que é repassado pelo professor e o que é assimilado pelo aluno, tornando o ensino monótono e desestimulante, favorecendo assim o desinteresse por parte do discente pela Química.

Os jogos têm o poder de transformar aulas comuns em momentos de um ensino eficiente, criativo e prazeroso para os alunos. Os jogos pedagógicos aliam o aprendizado de determinados conteúdos à atividade lúdica, despertando interesse dos alunos no assunto abordado e propiciando uma aprendizagem eficaz, divertida e empolgante (KISHIMOTO,1996; ROBAINA ,2008).
No campo educacional, uma das propostas que vêm subsidiando o ensinoaprendizagem em Ciências tem sido a resolução de problemas, pelo fato de se constituir um recurso que auxilia a construção de conceitos, procedimentos e atitudes relacionados a essa disciplina. É importante destacar como condição básica para utilizar com êxito atividades de resolução de problemas, no ensino de Ciências, - exercício da criatividade - capacidade fundamental para essa prática e que implica o surgimento de ideias novas e originais. (GARRET, 1988).

O ensino baseado na resolução de problemas está fundamentado no caráter contraditório do conhecimento, com o objetivo de que o estudante, como sujeito de aprendizagem, assimile os conteúdos e consiga refletir e resolver as diversas situações problemáticas. Dentro dessa perspectiva, foi escolhido o jogo Sudoku como método de ensino por problema.

Este trabalho, que agrega Matemática e Química, tem o objetivo de aplicar e avaliar o jogo didático (Sudokímica) por resolução de problemas e utilizar o recurso didático jogo como uma ferramenta facilitadora na transmissão do conhecimento; propor atividades com jogo, as quais levem o aluno a pôr em prática seus conhecimentos sobre tabela periódica, elementos químicos, entre outros; além de proporcionar ao aluno, com maior dificuldade de aprendizagem, uma nova chance de compreender e revisar o conteúdo estudado de forma mais agradável, incentivando a cooperação entre os alunos.

O Sudoku é um jogo projetado por Howard Garns, um arquiteto e construtor independente de quebra-cabeças que se baseou, provavelmente, no quadrado latino. Garns adicionou ao quadrado latino uma terceira dimensão e apresentou sua nova criação como uma grade parcialmente preenchida, em que o solucionador deveria preencher os demais quadros vazios.

\section{DESENVOLVIMENTO}

O Sudoku é uma mania mundial e tem como objetivo a colocação de números de 1 a 9 
em cada uma das células vazias numa grade de $9 \times 9$, constituída por $3 \times 3$ subgrades chamadas regiões. Cada coluna, linha e região só podem ter um número de 1 a 9 , como apresentado na Figura 1.

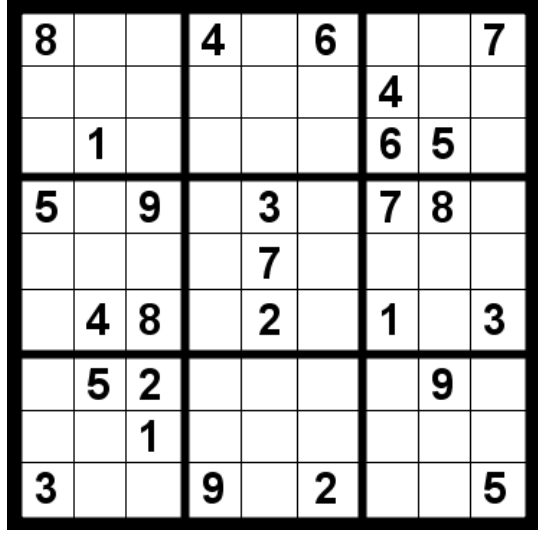

Figura 1. Sudoku tradicional

Esse jogo já tem conquistado muitos adeptos e sofrido várias alterações no seu conteúdo, mas manteve sempre o mesmo conceito. O Sudoku, além de ser uma diversão, desenvolve ainda o raciocínio lógico e a concentração, podendo por isso ser também usado na sala de aula. O jogo pode melhorar a capacidade de argumentação do aluno, já que, para colocar um número num espaço em branco, ele deve analisar e justificar mentalmente a sua jogada. (GRANDO, 2001). Tendo em vista essas características, o professor pode adaptar o Sudoku à disciplina de Química.

Como neste trabalho os números de 1 a 9 foram substituídos pelos símbolos dos elementos químicos, denominou-se 0 jogo adaptado de Sudokímica, fazendo com que o processo de ensino-aprendizagem seja o de reconhecer os símbolos dos elementos químicos da tabela periódica de forma lúdica e significativa.

O jogo Sudokímica foi aplicado em três turmas da Escola Estadual Professora Ana Júlia Mousinho, localizada na cidade de Natal, no estado do Rio Grande do Norte, totalizando noventa alunos. Para resolver o Sudokímica, os alunos utilizaram o site $^{1}$, no qual se pode jogar on-line. É possível jogar o Sudokímica através de um programa disponível gratuitamente na internet $^{2}$, conforme apresentado nas Figuras $2 \mathrm{e}$

$$
1
$$

21 Disponível em:
3.

A princípio, foram expostas, em sala de aula, a definição do Sudoku convencional, suas respectivas regras e a forma como o jogo foi adaptado para ensinar Química, ou seja, em vez de números de 1 a 9 serão colocados os símbolos dos elementos químicos. Foi discutido também como funciona o jogo on-line, já que nessa versão tem-se a contagem do tempo para o término do jogo. Em seguida, a turma encaminhou-se ao laboratório de informática para jogar o Sudokímica on-line.

O objetivo do jogo é completar o quadro com elementos químicos, de forma que não se repitam na diagonal, horizontal e vertical. O jogo tem o intuito de contribuir para que os alunos se tornem mais familiarizados com a tabela periódica e com os símbolos dos elementos químicos, como também de levar o aluno a desenvolver uma relação entre os nomes e os símbolos dos elementos da tabela periódica. $\mathrm{O}$ Sudokímica pode ser jogado individualmente ou em dupla em sala de aula.

Para dar início ao jogo, cada aluno ou dupla de alunos ficou em um computador e acessou o site, no qual está disponível o jogo on-line com um determinado tempo para concluir a tarefa.

Inicialmente, o professor pode auxiliar o aluno, no sentido de se desenvolver no jogo, já que nem todos conhecem e jogam o Sudoku. $O$ jogo somente termina quando um aluno completa todos os quadros, de modo que não se repita nenhum elemento do quadrado. A Figura 3 mostra a tela do jogo, com os símbolos dos elementos químicos, seguidos de seus respectivos nomes, bem como um tempo determinado para cumprir a tarefa.

Para jogar, o aluno deve clicar no elemento e arrastar até a posição desejada, sem repetir elementos na mesma linha ou coluna. Os quadros em um tom mais escuro são dados, para que exista somente uma resolução possível. Esses quadros não podem ser movidos. O aluno pode concluir o jogo mesmo sem completar o quadro, pressionando o botão "Terminar". Feito isso, os pontos serão

<http://www.cmdmc.com.br/sudoku/abre_jogo.php>. Disponível em:

<http://www.chemprofessor.com/ChemDoku/ChemDo ku.msi>.

PERIÓDICO TCHÊ QUÍMICA • www.periodico.tchequimica.com • Vol. 9 N. 17. • ISSN 1806-0374 (impresso) • ISSN 1806-9827 (CD-ROM) • ISSN 2179-0302 (meio eletrônico) 
calculados. O tempo e a porcentagem de elementos posicionados corretamente são fatores que alteram a pontuação. Vale ressaltar que cada jogo possui uma única resolução.

Como o jogo pode ser acessado on-line ou através de download de um software, tornouse necessária para o desenvolvimento dessa atividade apenas a utilização do laboratório de informática. Para as escolas que não têm laboratório de informática, é preciso somente imprimir o jogo em folha A4, como mostra a Figura 4.

\begin{tabular}{|c|c|c|c|c|c|c|c|c|c|}
\hline H & C & & $\mathbf{L i}$ & & & $\mathbf{F}$ & & & B \\
\hline $\mathrm{He}$ & & $\mathrm{He}$ & & & 0 & & & C & \\
\hline $\mathrm{Be}$ & 0 & & $\mathrm{~N}$ & $\mathrm{Be}$ & & & Li & & H \\
\hline Li & & & $\mathrm{He}$ & & & B & & & C \\
\hline B & & B & & & $\mathbf{F}$ & & & $\mathbf{N}$ & \\
\hline $\mathrm{C}$ & H & & & 0 & & & $\mathrm{He}$ & & \\
\hline $\mathrm{N}$ & & & 0 & & & Li & & & $\mathrm{He}$ \\
\hline 0 & & $\mathbf{N}$ & & & C & & & $\mathrm{Be}$ & \\
\hline$F$ & B & & & $\mathrm{He}$ & & & 0 & & \\
\hline
\end{tabular}

Figura 4. Jogo Sudokímica na versão impressa

Após a resolução do exercício, houve uma discussão no grande grupo, juntamente com o professor, sobre como chegaram a tais conclusões, momento em que o professor destacou as principais aplicações dos elementos encontrados.

\section{RESULTADOS E DISCUSSÃO}

Neste trabalho, buscou-se oferecer uma alternativa no ensino de Química aos alunos de uma escola estadual de ensino médio, aplicando o material didático. A aplicação do Sudokímica abrangeu cerca de 90 alunos, os quais demonstraram muita simpatia, motivação e interesse pelo jogo, apesar de, no início, alguns apresentarem dificuldades para jogar. Os alunos que já conheciam o jogo apresentaram maior facilidade em respondê-lo. Observou-se que o jogo despertou a curiosidade dos discentes, de uma forma prazerosa e estimulante, em relação aos nomes e aos símbolos dos elementos químicos da tabela periódica e suas respectivas aplicações, tornando a aprendizagem mais significativa.

O trabalho, através da aplicação do jogo Sudokímica, alcançou seu objetivo, visto que durante sua realização observou-se sua função lúdica e educativa, com o intuito de desenvolver estratégias importantes para o processo de ensino-aprendizagem de Química por resoluções de problemas, estimulando a motivação e facilitando a compreensão de conceitos complexos e abstratos e a interação entre alunos e professor.

Como as atividades lúdicas mobilizam esquemas mentais, elas estimulam o pensamento. Assim, quando o sujeito está diretamente envolvido na ação, fica mais fácil a compreensão do aspecto cognitivo, pois ocorre um desbloqueio mental. Esse fator é comprovado pelas observações do professor após a aplicação dos jogos, quando se verificou que os alunos que possuíam dificuldade com a matéria assimilaram mais facilmente o conteúdo, efetivando a aprendizagem e o interesse pela Química.

Dessa forma, o desenvolvimento desses jogos, corretamente criados e devidamente escolhidos com seus objetivos definidos, irá proporcionar aos alunos diversas interações, promovendo construções e aperfeiçoamentos de conceitos, habilidades e valorização do conhecimento, resgatando as lacunas que 0 processo de ensino-aprendizagem atual deixa em aberto e facilitando o pensamento e a construção do conhecimento.

O lúdico enquanto função educativa propicia a aprendizagem do educando, seu saber, sua compreensão de mundo e seu conhecimento. Sabe-se que o jogo é um fator didático de bastante relevância, e não apenas um passatempo, mas sim um elemento fundamental para o processo de ensinoaprendizagem. A educação pelo jogo deve, portanto, ser uma preocupação de todos os professores que têm intenção de motivar seus alunos ao aprendizado.

\section{CONCLUSÕES}

Concorda-se com vários autores que os aspectos lúdico e cognitivo presentes nos jogos 
são importantes estratégias para o ensino e a aprendizagem de conceitos abstratos e complexos. Porém, é importante lembrar que os jogos pedagógicos não são substitutos de outros métodos de ensino, mas sim um suporte para o professor e um poderoso motivador para que o aluno possa usufruir como recurso didático, facilitando a sua aprendizagem. Por outro lado, os professores precisam estar atentos aos objetivos da utilização de um jogo em sala de aula e saber como dar encaminhamento ao trabalho, após o seu uso. Além disso, devem dispor de subsídios que os auxiliem a explorar as possibilidades do jogo e a avaliar os seus efeitos em relação ao processo ensinoaprendizagem.

A utilização do jogo mostrou ser uma excelente alternativa para atuar como um elemento facilitador no processo ensinoaprendizagem, contribuindo também com a formação de alunos capazes de compreender as aplicações dos elementos químicos existentes no universo.

De uma forma geral, os jogos são um importante recurso para as aulas de Química, pois facilitam a aprendizagem do aluno, além de motivá-lo.

Conclui-se que o Sudokímica, sendo um jogo que estimula o raciocínio de investigação por resolução de problemas, quando associado ao ensino de Química, pode ser aplicado como material didático auxiliar/complementar facilitador no processo de ensino-aprendizagem de Química.

\section{AGRADECIMENTOS}

Aos alunos da E. E. Prof. Ana Júlia Mousinho (Natal-RN).

\section{REFERÊNCIAS}

1. Barros, P. M.; Silva, C. S.; Silva, A. C. S.; Jerônimo, D. D. Utilização de jogos didáticos no ensino de Química: dominó dos plásticos. In: CONGRESSO DE INICIAÇÃO CIENTÍFICA DA UNESP, 20., São José do Rio Preto, 03 a 09 de novembro de 2009. Disponível em: <http://prope.unesp.br/xxi_cic/27_335976 16852. pdf>. Acesso em: $\overline{3} 0$ out. 2011.
2. Garret, R. M. Resolución de problemas y creatividad: implicaciones para el currículo de ciências. Enseñanza de las Ciencias, Barcelona, v. 6, n. 3, p. 224230, 1988.

3. Godoi, T. A. F.; Oliveira, H. P. M.; Godognoto, L. Tabela periódica: um supertrunfo para alunos do ensino fundamental e médio. Química Nova na Escola, v. 32, n. 1, p. 22-25, 2010.

4. Grando, R. C. O jogo na educação: aspectos didático-metodológicos do jogo na educação matemática. 2001. Disponível <http://www.fae.unicamp.br/cempem/ lapemmec/cursos/el654/2001/juliana_e_cl audia/O_jogo_na_educacao.htm>. Acesso em 26 out. 2011.

5. Kishimoto, T. M. Jogo, Brinquedo, Brincadeira e a Educação. São Paulo: Cortez, 1996. 183p.

6. Mariscal, A. J. F.; Iglesias, M. J. Soletrando o Brasil com símbolos químicos. Química Nova na Escola, v. 31, n.1, p. 31-33, 2009.

7. Oliveira, L. M. S.; Silva, O. G; Ferreira, U. V. S. Desenvolvendo jogos didáticos para o ensino de química. Holos, Natal, ano 26, v. 5, n. 166, 2010.

8. Robaina, J. V. L. Química através do lúdico: brincando e aprendendo. Canoas: Ulbra, 2008. 480p.

9. Santos, A. F.; Fidelis, H. T.; Field's, K. A. P.; Felipe, L. M; Silva, L. T.; Santos, L. C.; Santos, R. A. Trilha química: uma inovação no processo ensinoaprendizagem. Intubiara: ULBRA, 2008.

10. Santos, A. P. B; Michel, R. C. Vamos jogar uma Suequímica. Química Nova na Escola, v. 31, n. 3, p. 179-183, 2009.

11. Vygotsky, L. S. A formação social da mente. São Paulo: Martins Fontes, 1989.

12. Watanabe, M.; Recena, C. P. R. Memória 
orgânica: um jogo didático útil no processo de ensino e aprendizagem. Universidade Federal de Mato Grosso do Sul, 2008.

13. Zanon, D. A. V.; Guerreiro, M. A. S.; Oliveira, R. C. Jogo didático Ludo Químico para o ensino de nomenclatura dos compostos orgânicos: projeto, produção, aplicação e avaliação. Ciências \& Cognição, Rio de Janeiro, v. 13, n. 1, p. 72-81, 2008.

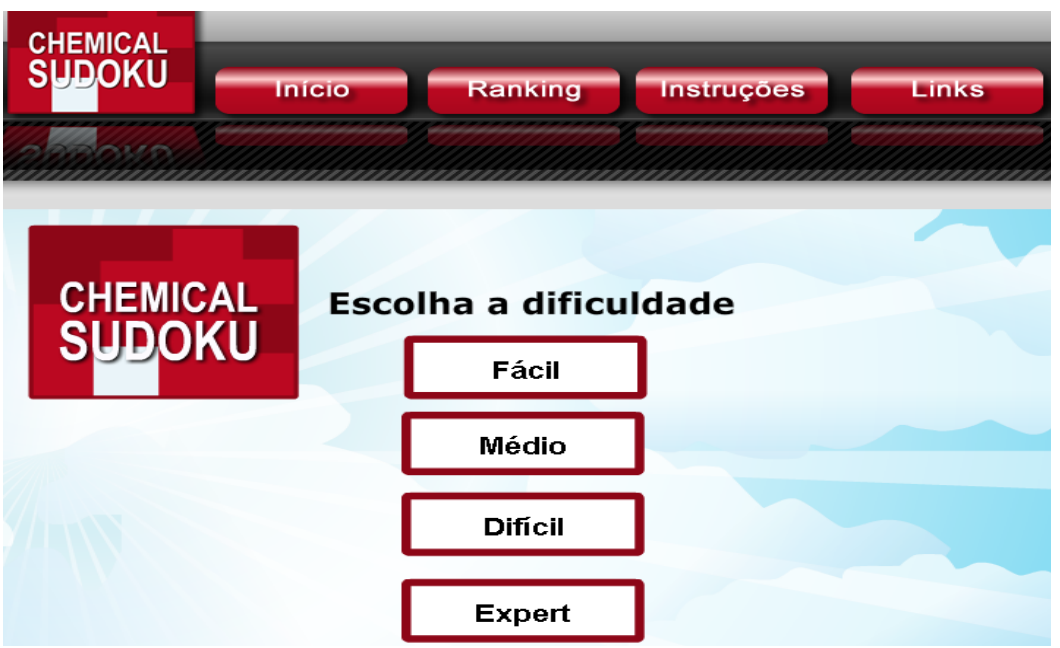

Figura 2. Níveis de dificuldade do Sudokímica on-line

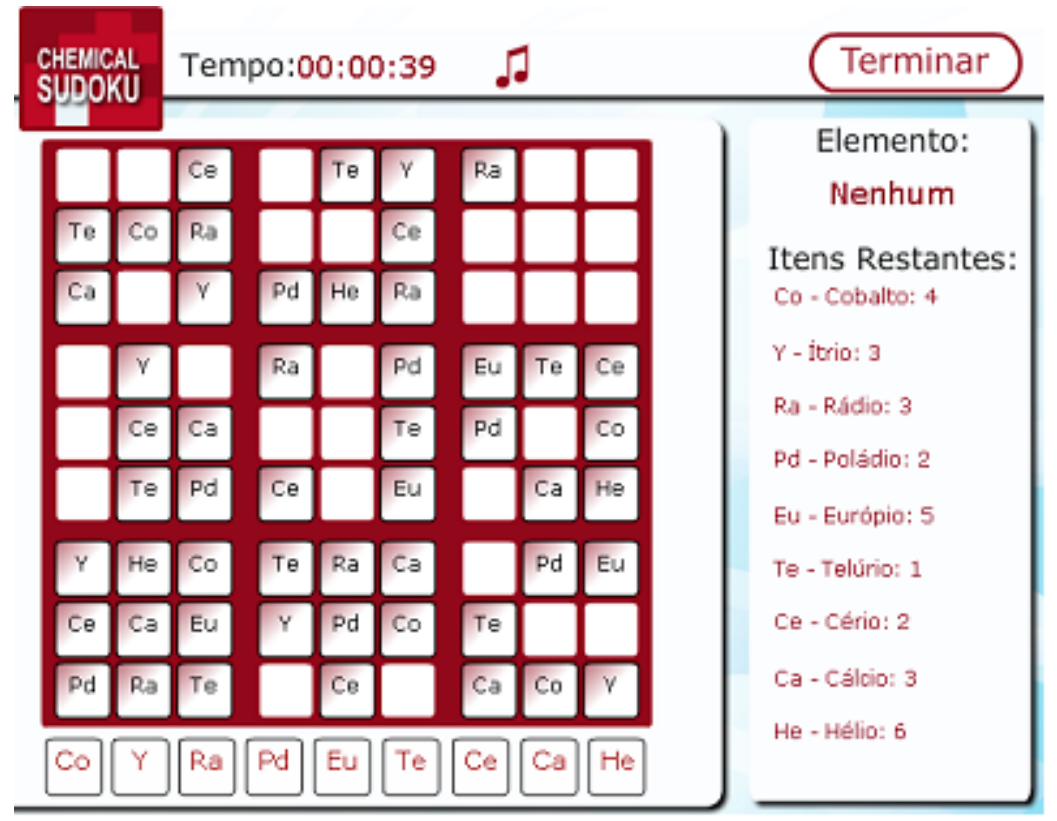

Figura 3. Sudokímica em execução no Chemical Sudoku

PERIÓDICO TCHÊ QUÍMICA • www.periodico.tchequimica.com • Vol. 9 N. 17. •ISSN 1806-0374 (impresso) • ISSN 1806-9827 (CD-ROM) • ISSN 2179-0302 (meio eletrônico)

(C) 2011. Porto Alegre, RS. Brasil

The Periódico Tchê Química (ISSN: 1806-0374; 2179-0302) is an open-access journal since 2004. Journal DOI: 10.52571/PTQ. http://www.tchequimica.com This text was introduced in this file in 2021 for compliance reasons.

๑) The Author(s)

OPEN ACCESS. This article is licensed under a Creative Commons Attribution 4.0 (CC BY 4.0) International License, which permits use, sharing, adaptation, distribution, and reproduction in any medium or format, as long as you give appropriate credit to the original author(s) and the source, provide a link to the Creative Commons license, and indicate if changes were made. The images or other third-party material in this article are included in the 作 